\title{
Associação do estado nutricional e aptidão cardiorrespiratória com a prática de atividade física e indicadores de obesidade familiar em escolares
}

\author{
Association of nutritional status and cardiorespiratory fitness with the practice of \\ physical activity and indicators of family obesity in schoolchildren
}

\section{AUTORES \\ Greice Graziela Moraes ${ }^{1}$ (D) \\ Debora Tornquist ${ }^{1}$ (D) \\ Luciana Tornquist ${ }^{1}$ (D) \\ Cézane Priscila Reuter ${ }^{2}$ (D) \\ Miria Suzana Burgos ${ }^{1,2}$ (D) \\ 1 Programa de Pós-Graduação em Promoção da Saúde. Universidade de Santa Cruz do Sul. Santa Cruz do Sul, Rio Grande do Sul, Brasil. \\ 2 Departamento de Educação Física e Saúde. Universidade de Santa Cruz do Sul. Santa Cruz do Sul, Rio Grande do Sul, Brasil.}

\section{CONTATO}

\section{Miria Suzana Burgos}

mburgos@unisc.br

Programa de Pós-Graduação em Promoção da Saúde - Universidade de Santa Cruz do Sul Av. Independência, 2293, Bloco 42, sala 4206, Bairro: Universitário, Santa Cruz do Sul, Rio Grande do Sul, Brasil. CEP: 96815-900.

DOI

10.12820/rbafs.v.22n6p540-45

\section{(6) $(\Theta \Theta \Theta$}

Copyright: This is an open-access article distributed under the terms of the Creative Commons Attribution License ${ }^{\circledR}$, which permits unrestricted use, distribution, and reproduction in any medium, provided that the original author and source are credited.

\begin{abstract}
RESUMO
Estudo teve como objetivo analisar a associação do índice de massa corporal (IMC) e aptidão cardiorrespiratória (APCR) com a prática de atividade física e indicadores de obesidade familiar em escolares. A amostra foi de 1.254 escolares de 7 a 17 anos de idade $(54,7 \%$ do feminino, $12,0 \pm 3,0$ anos). Avaliou-se o histórico de obesidade familiar, peso ao nascer, amamentação exclusiva por leite materno e prática de atividades físicas por meio de questionário. A APCR e IMC foram agrupados na variável APCR/IMC: 1) apto/eutrófico; 2) inapto/eutrófico; 3) apto/excesso de peso e; 4) inapto/ excesso de peso. Observou-se elevada proporção de escolares inaptos/obesos (19,7\% no masculino e $17,6 \%$ no feminino). APCR/IMC associou-se de forma negativa com a prática de atividades físicas [(RP= 0,80; IC95\%: 0,70-0,92) e $(\mathrm{RP}=0,73$; IC95\%: 0,58-0,91)]; e amamentação exclusiva $[(\mathrm{RP}=$ 0,82; IC95\%: 0,70-0,97) e ( $\mathrm{RP}=0,72$; IC95\%: 0,55-0,96)]; e de forma positiva com obesidade do pai $(\mathrm{RP}=2,20$; IC95\%: 1,43-3,40), e peso ao nascer $[(\mathrm{RP}=1,73$; IC95\%: 1,07-2,81) e $(\mathrm{RP}=2,52$; IC95\%: $1,06-5,99)]$. A prática de atividade física e amamentação exclusiva estão associadas a menor probabilidade e a obesidade do pai e o peso normal/elevado ao nascer a maior probabilidade de escolares apresentarem menores níveis de aptidão e excesso de peso.
\end{abstract}

Palavras-chave: Sobrepeso; Obesidade; Aptidão física; Escolares.

\section{ABSTRACT}

The objective of this study was to analyze the association between body mass index (BMI) and cardiorespiratory fitness (APCR) with physical activity and indicators of family obesity in schoolchildren. The sample consisted of 1.254 schoolchildren aged 7 to 17 years (54,7\% female, 12,0 \pm 3,0 years). We evaluated the bistory of family obesity, birth weight, exclusive breastfeeding for breast milk and physical activity practice through a questionnaire. The APCR and BMI were grouped in the variable APCR/IMC: 1) aptleutrophic; 2) unfit/eutrophic; 3) fitloverweight and; 4) unfit/overweight. A high proportion of unfit/obese students was observed $(19,7 \%$ in males and $17,6 \%$ in females). APCR / IMC was negatively associated with physical activity (PR=0,80; 95\%CI: 0,70-0,92) and (PR=0,73; 95\%CI: 0,58-0,91); and exclusive breastfeeding [(PR= 0,82; 95\%CI: 0,70-0,97) and (PR=0,72; 95\%CI: 0,55-0,96)]; $(O R=2,20 ; 95 \% C I: 1,43-3,40)$, and birth weight $(R P=1,73 ; 95 \% C I: 1,07-2,81)$ and ( $R P=2,52 ; 95 \% C I: 1,06-5,99)]$. The practice of physical activity and exclusive breastfeeding are associated with a lower probability and the father's obesity and the normal/high weight at birth are more likely to present lower levels of aptitude and excess weight.

Keywords: Overweight; Obesity; Physical fitness; Schoolchildren.

\section{Introdução}

Os elevados índices de obesidade ${ }^{1,2}$ e de baixos níveis de aptidão cardiorrespiratória $(\mathrm{APCR})^{3} \mathrm{em}$ crianças e adolescentes já são bem evidenciados. No Brasil, a prevalência de sobrepeso e obesidade na população infanto-juvenil também é elevada, em especial na região sul $(25,7 \% \text { para sobrepeso e } 10,4 \% \text { para obesidade })^{4}$.
Baixos níveis de APCR têm sido observados em 50,8\% de crianças e adolescentes ${ }^{5}$. Além disso, a incidência de excesso de peso e baixos níveis de APCR apresentam crescente aumento em escolares do sul do Brasil ${ }^{6}$.

A relação entre a presença de excesso de peso corporal e baixos níveis de APCR, de forma conjunta, foi pouco explorada na literatura. Estudo demonstrou que 
adolescentes com IMC adequado, mas com menores níveis de APCR, apresentaram melhor perfil de risco cardiometabólico, em comparação aos seus pares com IMC elevado, mas com bons níveis de APCR. Esses achados sugerem que manter o IMC adequado seja mais importante para o perfil cardiometabólico, do que apresentar melhores níveis de $\mathrm{APCR}^{7}$. Desse modo, o IMC parece moderar a associação entre APCR e a presença de risco cardiometabólico em escolares ${ }^{8}$.

Outros estudos ${ }^{9,10}$ sugerem que adolescentes obesos com níveis mais elevados de APCR são metabolicamente mais saudáveis comparados aos obesos com perfil metabólico alterado. Em crianças e adolescentes brasileiros foi observado que a presença de dislipidemia é mais frequente nos escolares com baixos níveis de APCR e com excesso de peso ${ }^{11}$. Porém, a presença de apenas um componente alterado (excesso de peso ou inaptidão cardiorrespiratória) não esteve associado com a presença de dislipidemia ${ }^{11}$.

Diante das lacunas previamente descritas, em especial na utilização da forma de avaliação conjunta dos níveis de APCR e estado nutricional, este estudo teve como objetivo analisar a associação entre o estado nutricional e APCR com a prática de atividade física e indicadores de obesidade familiar em escolares.

\section{Métodos}

Estudo transversal, que teve como população alvo 20.540 escolares do município de Santa Cruz do Sul, Rio Grande do Sul, do ensino Fundamental e Médio, divididos em 69 escolas da rede pública (estaduais e municipais) e privada do município. Destas, 19 escolas (14 escolas da zona urbana e 5 da zona rural), foram selecionadas aleatoriamente para o estudo, por meio de sorteio, respeitando a densidade populacional de escolares por regiões, estratificadas por conglomerados (norte, sul, leste, oeste e centro), abrangendo a zona urbana e rural.

Nas escolas selecionadas, foram convidados os alunos na faixa etária de sete a 17 anos para participar do estudo, através do envio aos pais de uma carta explicando os objetivos e procedimentos da pesquisa, anexo a duas vias do termo de consentimento livre e esclarecido (TCLE). Os alunos que retornaram com o TCLE assinado pelos pais ou responsáveis para a escola, autorizando a participação de seus filhos no estudo, foram incluídos na pesquisa. A pesquisa foi aprovada pelo Comitê de Ética em Pesquisa com Seres Humanos da Universidade de Santa Cruz do Sul (CEP - UNISC - Processo 2959-11). As coletas de dados foram realizadas nos anos de 2011 e 2012.
As medidas de peso e estatura foram realizadas em balança antropométrica mecânica Filizola, por avaliador treinado, sendo realizada uma medida por aluno, estando este descalço e utilizando o mínimo de roupas possível. Foram observadas as recomendações do Projeto Esporte Brasil (PROESP-BR) para avaliação ${ }^{12}$. Posteriormente, foi calculado índice de massa corporal - IMC e classificado de acordo com os critérios do CDC/NCHS ${ }^{13}$ : baixo peso: $<\mathrm{p} 5$, normal: $\geq \mathrm{p} 5$ e $<\mathrm{p} 85$, sobrepeso: $\mathrm{p} \geq 85$ e $<$ p95 e; obesidade $\geq$ p95.

$\mathrm{A}$ aptidão cardiorrespiratória (APCR) foi avaliada por meio do teste de pista de corrida e caminhada de 9 minutos (PROESP-BR ${ }^{12}$ ) e categorizada em: apto, aqueles com valores em níveis de normalidade para os pontos de corte por sexo e idade do PROESP-BR ${ }^{12}$, e inapto, cujo valores ficaram na zona de risco destes mesmos pontos de corte. Anteriormente à realização dos testes, os escolares foram instruídos a utilizarem roupa leve e tênis para a realização do teste.

A variável APCR/IMC foi definida conforme segue: 1) aptos/eutróficos: escolares aptos e com IMC normal (eutróficos); 2) aptos/excesso de peso: escolares aptos e que apresentaram sobrepeso ou obesidade; 3 ) inaptos/eutróficos: escolares inaptos (aptidão física insatisfatória) e com IMC normal; 4) inaptos/excesso de peso: escolares inaptos e com sobrepeso ou obesidade.

Foram consideradas recusas os escolares que não retornaram o termo de consentimento, e perdas os escolares que não compareceram no dia da coleta de dados agendada, que não preencheram corretamente o instrumento de coleta ou que não puderam realizar alguma das avaliações.

As demais variáveis do estudo foram coletadas por meio de questionário adaptado de Barros e $\mathrm{Nahas}^{14}$. A prática de atividade física foi referida pelos escolares ( parte da rotina típica do escolar e sendo desconsideradas atividades que o escolar relatava realizar apenas ocasionalmente. Não foram levadas em consideração a duração e frequência semanal das atividades relatadas.

O histórico de obesidade familiar e amamentação exclusiva por leite materno foram referidos pelos pais (sim ou não). Em relação a variável peso ao nascer, os pais respondiam o peso exato ou aproximado do filho no momento do nascimento e, posteriormente, os escolares foram classificados em três categorias: baixo peso (menos de 2999g), peso normal (3000g a 3999g) e peso elevado ( $4000 \mathrm{~g}$ ou mais valores) $)^{15}$.

Para a análise estatística, utilizou-se o programa SPSS for Windows 23.0 (IBM, NY, EUA). Foi aplica- 
do o teste de Shapiro-Wilk para avaliar a aderência dos dados à distribuição normal. As medidas de estatística descritiva (frequências e percentuais e médias e desvio padrão) foram utilizadas para a descrição das variáveis. Para comparação das médias de idade, peso, estatura, IMC e APCR entre os sexos foi realizada por meio do teste t de Student para dados independentes. A regressão de Poisson, com variância robusta, foi aplicada para testar a associação da variável APCR/IMC com a prática de atividades físicas, histórico familiar de obesidade, peso ao nascer e amamentação exclusiva do escolar. As razões de prevalência brutas e ajustadas, e seus respectivos intervalos de confianças de 95\% (IC95\%) para expressar os resultados das associações. $\mathrm{Na}$ análise ajustada foram incluídas no modelo as variáveis sexo, idade e classe econômica, mensurada através do critério $\mathrm{ABEP}^{16}$. Foram criados três modelos, em todos, a variável APCR/IMC foi tratada como desfecho e a categoria apto/eutrófico como sendo a de referência. Para todos os testes utilizados foi adotado nível de significância de $\mathrm{p}<0,05$.

\section{Resultados}

A amostra do estudo foi composta por 1.254 escolares de 7 a 17 anos (54,7\% do feminino, 12,0 \pm 3,0 anos). $\mathrm{Na}$ tabela 1, são apresentadas a média de idade, das variáveis antropométricas e de aptidão cardiorrespiratória da amostra de escolares de Santa Cruz do Sul, que participaram do estudo, estratificadas por sexo. Bem como o percentual de escolares em cada zona de classificação da variável APCR/IMC, na qual verificou-se que nos escolares do sexo masculino, 13,6\% foram classificados como aptos/excesso de peso, 26,4\% como inapto/eutrófico e $18,7 \%$ estavam acima do peso e fora da zona recomendada para a APCR (inapto/excesso de peso). Nos do sexo feminino, 37,3\% foram classificadas como inaptas/eutróficas; 8,0\% apresentaram excesso de peso e APCR satisfatória (apta/excesso de peso) e 17,6\% apresentaram a APCR abaixo dos níveis recomendados (inapta/excesso de peso).

Os resultados das associações entre a variável APCR/IMC com a prática de atividade física e os indicadores de obesidade familiar, peso ao nascer e amamentação são apresentados na Tabela 2. A APCR/ IMC associou-se de forma negativa e significativa com a prática de atividades físicas [inaptidão/eutrofia $(\mathrm{RP}=$ 0,80; IC95\%: 0,70-0,92) ou inaptidão/excesso de peso $(\mathrm{RP}=0,73$; IC95\%: 0,58-0,91)]; e com a amamentação exclusiva por leite materno até os seis meses [inaptidão/ eutrofia ( $\mathrm{RP}=0,82$; IC95\%: 0,70-0,97) e inaptidão/ex-
Tabela 1 - Características antropométricas e da aptidão cardiorrespiratória da amostra de escolares de Santa Cruz do Sul, Rio Grande do Sul, 2011-2012 ( $\mathrm{n}=1.254)$.

\begin{tabular}{lccc}
\hline \multirow{2}{*}{ Variáveis } & Masculino & Feminino & \multirow{2}{*}{ Valor p } \\
\cline { 2 - 3 } & Média $\pm \mathrm{DP}$ & Média $\pm \mathrm{DP}$ & \\
\hline Idade (anos) & $11,7 \pm 3,0$ & $12,0 \pm 3,0$ & 0,598 \\
Peso corporal $(\mathrm{kg})$ & $47,9 \pm 16,3$ & $46,3 \pm 14,1$ & $<0,001$ \\
Estatura $(\mathrm{cm})$ & $152,5 \pm 17,2$ & $150,4 \pm 13,5$ & $<0,001$ \\
$\mathrm{IMC}^{*}\left(\mathrm{~kg} / \mathrm{m}^{2}\right)$ & $20,0 \pm 3,8$ & $20,0 \pm 3,9$ & 0,811 \\
APCR $^{*}(\mathrm{~m})$ & $1415,5 \pm 301,3$ & $1170,9 \pm 201,7$ & $<0,001$ \\
& $\mathrm{n}(\%)$ & $\mathrm{n}(\%)$ & \\
Interação entre APCR/IMC & & & \\
Apto/Eutrófico & $229(40,3)$ & $256(37,2)$ & \\
Apto/Excesso de peso & $77(13,6)$ & $55(8,0)$ & $<0,001$ \\
Inapto/Eutrófico & $150(26,4)$ & $254(37,0)$ & \\
Inapto/Excesso de peso & $112(19,7)$ & $121(17,6)$ & \\
\hline
\end{tabular}

$\mathrm{IMC}=$ índice de massa corporal; $\mathrm{APCR}=$ aptidão cardiorrespiratória.

Tabela 2 - Razão de prevalência entre o estado nutricional e aptidão física com indicadores de atividade física e obesidade familiar em escolares de Santa Cruz do Sul, Rio Grande do Sul, 2011-2012 (n=1.254).

\begin{tabular}{|c|c|c|c|}
\hline & $\begin{array}{l}\text { Apto/Excesso de } \\
\text { peso }\end{array}$ & $\begin{array}{l}\text { Inapto/ } \\
\text { Eutrófico }\end{array}$ & $\begin{array}{c}\text { Inapto/excesso } \\
\text { de peso }\end{array}$ \\
\hline & RP (IC 95\%) & RP (IC 95\%) & RP (IC 95\%) \\
\hline \multicolumn{4}{|l|}{ Atividade física } \\
\hline Não pratica & 1 & 1 & 1 \\
\hline Pratica & $0,98(0,92-1,04)$ & $0,80(0,70-0,92)$ & $0,73(0,58-0,91)$ \\
\hline \multicolumn{4}{|l|}{ Obesidade do pai } \\
\hline Pai eutrófico & 1 & 1 & 1 \\
\hline Pai obeso & $1,04(0,89-1,22)$ & $0,87(0,59-1,26)$ & $2,20(1,43-3,40)$ \\
\hline \multicolumn{4}{|l|}{ Obesidade da mãe } \\
\hline Mãe eutrófica & 1 & 1 & 1 \\
\hline Mãe obesa & $1,08(0,97-1,21)$ & $0,99(0,75-1,32)$ & $1,07(0,69-1,66)$ \\
\hline \multicolumn{4}{|c|}{ Obesidade da avó materna } \\
\hline Avó eutrófica & 1 & 1 & 1 \\
\hline Avó obesa & $0,90(0,81-1,00)$ & $0,93(0,70-1,23)$ & $1,26(0,81-1,95)$ \\
\hline \multicolumn{4}{|c|}{ Obesidade do avô materno } \\
\hline Avô eutrófico & 1 & 1 & 1 \\
\hline Avô obeso & $1,13(0,99-1,30)$ & $1,23(0,89-1,70)$ & $1,23(0,74-2,04)$ \\
\hline \multicolumn{4}{|l|}{ Peso ao nascer } \\
\hline Baixo peso & 1 & 1 & 1 \\
\hline Peso normal & $1,13(1,00-1,28)$ & $1,18(0,84-1,64)$ & $1,73(1,07-2,81)$ \\
\hline Peso elevado & $1,22(0,98-1,51)$ & $1,31(0,76-2,27)$ & $2,52(1,06-5,99)$ \\
\hline \multicolumn{4}{|c|}{ Amamentação com leite materno* } \\
\hline Não & 1 & 1 & 1 \\
\hline Sim & $0,94(0,88-1,02)$ & $0,82(0,70-0,97)$ & $0,72(0,55-0,96)$ \\
\hline
\end{tabular}

Análise ajustada para sexo, idade e classe econômica; RP: razão de prevalência; IC: intervalo de confiança de 95\%; *exclusivo até os seis meses.

cesso de peso (RP= 0,72; IC95\%: 0,55-0,96)]. Também, observou-se associação significativa e positiva entre APCR/IMC e obesidade do pai [inaptidão/excesso de 
peso $(\mathrm{RP}=2,20$; IC95\%: 1,43-3,40)], e o peso ao nascer [peso normal ( $R P=1,73$; IC95\%: 1,07-2,81) e elevado ao nascer ( $\mathrm{RP}=2,52$; IC95\%: 1,06-5,99). A obesidade da mãe, da avó ou avô materno não apresentaram associações significativas com a variável APCR/IMC.

\section{Discussão}

Os resultados do presente estudo demonstram que a variável APCR/IMC dos escolares se associou positivamente com a obesidade do pai e o peso ao nascer do escolar, e negativamente com a prática de atividades físicas e amamentação exclusiva por leite materno até os seis meses.

Os escolares que relataram praticar atividades físicas apresentavam menor propensão a baixos níveis de inaptidão e excesso de peso corporal. Já está bem evidenciado na literatura que a prática de exercícios físicos auxilia na melhora dos níveis de aptidão cardiorrespiratória ${ }^{17}$ e também na redução do $\mathrm{IMC}^{18}$. Isso reforça a importância de se incentivar a prática de exercícios físicos, especialmente na população de crianças e adolescentes, tendo em vista que níveis insatisfatórios de $\mathrm{IMC}^{3,5,7,11}$ e de APCR ${ }^{3,5,7,11,19}$ têm sido associados com outros fatores de risco para doenças cardiovasculares. Moschoniset al. ${ }^{7}$ verificaram que os escolares com IMC adequados, mas com baixos níveis de APCR, tinham melhor perfil de risco cardiometabólico comparados aos escolares com IMC elevado e bons níveis de APCR. Estes resultados reforçam a importância de manter o IMC adequado, independente dos níveis de APCR do escolar. Díez-Fernández et $\mathrm{al}^{8}$, sugerem que o IMC tem papel de mediação entre os níveis de APCR a presença de risco cardiometabólico em escolares. Para Eisenmann ${ }^{19}$, evidências apontam que a manutenção de uma boa aptidão cardiorrespiratória pode atenuar os componentes da síndrome metabólica em obesos, sendo que aptos/excesso de peso possuem menos fatores de risco do que os inaptos/eutróficos.

Escolares que receberam amamentação exclusiva por leite materno até os seis meses apresentaram menores níveis inaptidão e excesso de peso corporal. $\mathrm{O}$ aleitamento materno é observado como fator de proteção a obesidade infantil ${ }^{20,21}$. No entanto, não foram encontrados estudos que investigassem a associação da amamentação com a variável APCR/IMC, como no presente estudo, ou com a aptidão cardiorrespiratória. Levanta-se a hipótese de que o leite materno possa influenciar na aptidão física dos escolares por propiciar um desenvolvimento e crescimento adequados, que a médio e/ou longo prazo possa também auxiliar na manutenção de níveis satisfatórios desta variável.
No que se refere o histórico familiar de obesidade, observou-se maior probabilidade de escolares cujo pai era obeso apresentarem inaptidão/excesso de peso. Porém, a obesidade da mãe, da avó ou avô materno não se associaram a variável APCR/IMC. Em relação a associação entre APCR/IMC e obesidade paterna identificada no presente estudo, cabe salientar que o comportamento das crianças depende muito do ambiente familiar, e não é possível analisar o nível de atividade física, sem considerá-lo ${ }^{22}$. Estudo ${ }^{23}$ com 557 crianças e 517 adolescentes suecos, identificou que o sobrepeso dos pais foi determinante para sobrepeso/obesidade em crianças e adolescentes estudados, reforçando achados do presente estudo. Jovens cujos pai e mãe apresentam excesso de peso ou obesidade demostraram três vezes maior probabilidade de estarem acima do peso e terem uma circunferência da cintura de alto risco, em comparação com aqueles cujos pais eram eutróficos, os autores também alegam serem os hábitos alimentares, passados dos pais para os filhos, o determinante desta associação ${ }^{23}$.

Apesar de a relação entre a presença de obesidade familiar e a obesidade do escolar esta bem documentada, a analisar da exposição simultânea ao excesso de peso e baixos níveis de APCR ainda são escassos na literatura. Não foram encontrados estudos que associando a APCR de crianças e adolescentes com o histórico de obesidade familiar. Porém, levanta-se a hipótese de que escolares que apresentem um histórico familiar de obesidade tenham a tendência a apresentar um estilo de vida mais inativo, uma vez que adultos obesos tendem a praticar menos atividades físicas e passar mais tempo atividades sedentárias ${ }^{24}$. Corroborando a hipótese levantada, estudo de Raudsepp ${ }^{25}$ encontrou associação positiva entre a atividade física de adolescentes e incentivo familiar à prática esportiva, sendo o exemplo passado dentro de casa uma das maneiras deste incentivo. Considerando que os níveis de atividade física influenciam a APCR dos escolares ${ }^{17}$, esta poderia ser uma possível suposição da associação encontrada entre as variáveis.

O risco metabólico de escolares tem demonstrado associação com a obesidade familiar. Escolares que a mãe é obesa tem maior probabilidade de apresentar risco metabólico e, ainda maior, se o pai e a mãe forem obesos $^{5}$. Ainda, escolares que apresentam baixa aptidão cardiorrespiratória e mãe obesas tem maior chance de apresentarem excesso de peso ${ }^{6}$.

Os escolares que ao nascer apresentavam peso normal ou elevado apresentaram maior probabilidade de ser inaptidão/excesso de peso. Embora não sejam encontrados na literatura estudos investigando a associa- 
ção observada entre APCR/IMC e o peso ao nascer dos escolares, observa-se uma contradição entre os estudos que buscam associar o peso ao nascer ao estado nutricional de crianças e adolescentes, em que, segundo dados de uma revisão sistemática, tanto o baixo peso ao nascer, quanto o elevado peso ao nascer vem sendo associados ao desenvolvimento de excesso de peso corporal, embora o elevado peso ao nascer apareceu associado ao sobrepeso/obesidade na maioria dos artigos. Os autores relatam que o nível socioeconômico tem sido apontado como um importante variável de confusão nesta associação $^{26}$. No que se refere a associação da APCR com o peso ao nascer, os estudos existentes na literatura apontam que escolares que nasceram com baixo peso apresentam resultados inferiores de capacidade aeróbica do que aqueles que nasceram com peso normal ${ }^{27,28}$.

Este estudo apresenta como pontos fortes: a abordagem utilizada, unindo as variáveis APCR e IMC, pouco explorada na literatura, principalmente no Brasil; considerou diversos indicadores de obesidade familiar, peso ao nascer e histórico de amamentação; envolveu uma amostra representativa de escolares de um município do sul do Brasil.

Por outro lado, há limitações que precisam ser consideradas na interpretação dos achados: o estudo apresenta diversas variáveis referidas pelos escolares (prática de atividade física) e pelos pais/responsáveis (obesidade familiar, peso ao nascer, amamentação), ficando suscetível à viés de informação e/ou de memória e; o delineamento transversal não permite estabelecer relações causais entre as variáveis analisadas.

Conclui-se que a prática de atividade física e a amamentação exclusiva por leite materno até os seis meses se mostraram associadas a menor probabilidade de os escolares apresentarem menores níveis de aptidão e excesso de peso. Por outro lado, a obesidade do pai e o peso normal ou elevado ao nascer se associou a maior probabilidade de os escolares apresentarem níveis insatisfatórios de aptidão e excesso de peso.

\section{Conflito de interesses}

Os autores declaram não haver conflito de interesses.

\section{Contribuição dos autores}

Moraes GG, participou da concepção inicial do artigo. Tornquist $\mathrm{D}$ e Tornquist $\mathrm{L}$ foram responsáveis pela coleta dos dados. Reuter CP foi responsável pela análise estatística dos dados. Burgos MS foi coordenadora do projeto. Todos os autores participaram da redação e revisão crítica do artigo, tendo aprovado a sua versão final.

\section{Referências}

1. Tsujimoto T, Kajio H, Sugiyama T. Obesity, diabetes, and length of time in the United States: Analysis of National Health and Nutrition Examination Survey 1999 to 2012. Medicine. 2016;95(35):1-7.

2. Ahluwalia N, Dalmasso P, Rasmussen M, Lipsky L, Currie $\mathrm{C}, \mathrm{Haug} \mathrm{E}$, et al. Trends in overweight prevalence among 11-, 13 - and 15-year-olds in 25 countries in Europe, Canada and USA from 2002 to 2010. Eur J Public Health. 2015;25:28-32.

3. Santos FK, Prista A, Gomes TN, Santos D, Damasceno A, Madeira A, et al. Body mass index, cardiorespiratory fitness and cardiometabolic risk factors in youth from Portugal and Mozambique. Int J Obes. 2015;39(10):1467-74.

4. Niehues JR, Gonzales AI, Lemos RR, Bezerra PP, Haas P. Prevalence of overweight and obesity in children and adolescents from the age range of 2 to 19 years old in Brazil. Int J Pediatr. 2014;2014:583207.

5. Todendi PF, Valim AR, Reuter CP, Mello ED, Gaya AR, Burgos MS. Metabolic risk in schoolchildren is associated with low levels of cardiorespiratory fitness, obesity, and parents' nutritional profile. J Pediatr. 2016;92(4):388-93.

6. Gaya AR, Reuter CP, Reuter ÉM, Franke SIR, Prá D, Gaya ACA,et al. Cumulative incidence of youth obesity is associated with low cardiorespiratory fitness levels and with maternal overweight. Motriz. 2015;21(4):407-14.

7. Moschonis G, Mougios V, Papandreou C, Lionis C, Chrousos GP, Malandraki E, et al. "Leaner and less fit" children have a better cardiometabolic profile than their "heavier and more fit" peers: the Healthy Growth Study. Nutr Metab Cardiovasc Dis. 2013;23(11):1058-65.

8. Díez-Fernández A, Sánchez-López M, Mora-Rodríguez R, Notario-Pacheco B, Torrijos-Niño C, Martínez-Vizcaíno V. Obesity as a mediator of the influence of cardiorespiratory fitness on cardiometabolic risk: a mediation analysis. Diabetes Care. 2014;37(3):855-62.

9. Ortega FB, Cadenas-Sánchez C, Sui X, Blair SN, Lavie CJ. Role of fitness in the metabolically healthy but obese phenotype: a review and update. Prog Cardiovasc Dis. 2015;58(1):76-86

10. Sénéchal M, Wicklow B,Wittmeier K, Hay J, MacIntosh AC, Eskicioglu P, et al. Cardiorespiratory fitness and adiposity in metabolically healthy overweight and obese youth. Pediatrics. 2013;132(1):e85-92.

11. Reuter CP, Silva PT, Renner JD, Mello ED, Valim AR, Pasa L, et al. Dyslipidemia is associated with unfit and overweight-obese children and adolescents. Arq Bras Cardiol.2016;106(3):188-93.

12. Projeto Esporte Brasil - PROESP-BR. Manual de aplicação de medidas de testes somatomotores. Porto Alegre: Universidade Federal do Rio Grande do Sul - UFRGS, 2009.

13. Centers for Disease Control and Prevention/ National Center for Health Statistics. CDC Growth Charts: United States. 2000. Available from: http://www.cdc.gov/growthcharts.

14. Barros MVG, Nahas MV. Medidas da atividade física: teoria e aplicação em diversos grupos populacionais. Londrina: Midiograf; 2003.

15. Puffer RR, Serrano CV. Patterns of birthweights. Washington (DC): Pan American Health Organization; 1987.

16. Associação Brasileira de Empresas de Pesquisa - ABEP. Critério de classificação econômica Brasil. 2012. Available from: http://www.abep.org/criterio-brasil. Dados do acesso.

17. American College of Sports Medicine - ACSM. Guidelines for Exercise Testing and Prescription. 2006. 7th edition. Philadelphia: Lippincott Williams \& Wilkins. 
18. World Health Organization - WHO. Obesity: Preventing and Managing the Global Epidemic. Geneva, 2000. In: World Health Organ Tech Rep Series, v. 894.

19. Eisenmann JC. Aerobic fitness, fatness and the metabolic syndrome in children and adolescents. Acta Paediatr. 2007;96(12):1723-9.

20. Simon VGN, Souza JMP, Souza S B. Aleitamento materno, alimentação complementar, sobrepeso e obesidade em préescolares. Rev Saúde Pública. 2009;43(1):60-9.

21. Arenz S, Rückerl R, Koletzko B, Von Kries R. Breastfeeding and childhood obesity- a systematic review. Int J Obes Relat Metab Disord. 2004;28(10):1247-56.

22. Sánchez-Cruz JJ, de Ruiter I, Jiménez-Moleón JJ. Individual, family and environmental factors associated with pediatric excess weight in Spain: a cross-sectional study. BMC Pediatr. 2014;14:3.

23. Ortega FB, Ruiz JR, Sjostrom M. Physical activity, overweight and central adiposity in Swedish children and adolescents: the European Youth Heart Study. Int J Behav Nutr Phys Act. 2007;4:61.

24. Rosique-Esteban N, Díaz-López A, Martínez-González MA, Corella D, Goday A, Martínez JA, et al. Leisuretime physical activity, sedentary behaviors, sleep, and cardiometabolic risk factors at baseline in the PREDIMEDPLUS intervention trial: A cross-sectional analysis. PLoSOne. 2017;12(3):e0172253.
25. Raudsepp L. The relationship between socio-economic status, parental support and adolescent physical activity. Acta Paediatr. 2006;95(1):93-8.

26. Rossi C E, Vasconcelos F A G. Peso ao nascer e obesidade em crianças e adolescentes: uma revisão sistemática. Rev Bras Epidemiol. 2010;13(2): 246-58.

27. Smith LJ, Van Asperen PP, McKay KO, Selvadurai H, Fitzgerald DA. Reduced exercise capacity in children born very preterm. Pediatrics. 2008;122(2):287-93.

28. Kriemler S, Keller H, Saigal S, Bar-Or O. Aerobic and lung performance in premature children with and without chronic lung disease of prematurity. Clin J Sport Med. 2005;15(5):349-55.

Recebido: 27/03/2016 Aprovado: 16/08/2017

\section{Como citar este artigo:}

Moraes GG, Tornquist D, Tornquist L, Reuter CP, Burgos MS. Associação do estado nutricional e aptidão cardiorrespiratória com a prática de atividade física e indicadores de obesidade familiar em escolares. Rev Bras Ati Fis Saúde. 2017;22(6):540-45. DOI: 10.12820/rba-

$$
\text { fs. v.22n6p540-45. }
$$

